\title{
Antecedent Contained Deletion and Pied-Piping: Evidence for a Variable-Free Semantics*
}

\author{
Pauline Jacobson \\ Brown University
}

\section{Introduction}

This paper explores some (as far as I know) previously unobserved interactions of Antecedent Contained Deletion (ACD) and Pied-Piping. Although the facts seem quite puzzling at first glance, I show that they fall neatly into place given three independently motivated proposals: (1) the analysis of VP Ellipsis in Rooth (1992); (2) the machinery needed in order to maintain a variable-free semantics (Jacobson, 1992b, 1996, to appear); and (3) the analysis of ACD as Transitive Verb Phrase (TVP) Ellipsis (Cormack, 1985; Jacobson, 1992a, 1992b). (The analysis of ACD as TVP ellipsis is itself rooted in a variable-free account of extraction.)

A word about the more general implications. By a "variable-free semantics", I mean one which makes no use of variables - and hence al so no use of assignment functions - as part of the semantic machinery. In a series of papers (see especially Jacobson, to appear), I have argued that this allows for surface structures to be directly assigned a model-theoretic interpretation without being mapped into a mediating level of LF. (This thus allows the compositional semantics to work directly in tandem with the syntactic combinatorics.) For example, certain interactions of extraction and pronominal binding which are of ten taken to necessitate a level of LF at which extracted material is "reconstructed" into the position of a gap turn out under this view of binding to be perfectly compatible with surface compositionality. (The variable-free program also dispenses with indices and traces in the syntax.) And, independent of the issue of surface compositionality, I have argued that there is considerable additional motivation for the variable-free semantics. Nonetheless, it is reasonable to ask whether the machinery needed to maintain a variable-free semantics represents a significant complication over the standard view of binding. In Jacobson (to appear) I address this question in detail, arguing of course that it does not. The present paper adds one new reason to believe that the requisite tools involve no new complications: we will see that the interactions of ACD and Pied-Piping provide striking conf irmation for one of the pieces of machinery needed to maintain variable-free semantics (the $\mathrm{g}$ rule, to be discussed below).

Before continuing, I should point out that Heim (1997) examines a related puzzle concerning ACD (from Kennedy, 1994) and arrives at the opposite conclusion. That is, she argues for a theory of semantics which makes more robust use of assignment functions (and hence variables) than does the standard semantics. I will not have space to thoroughly discuss this puzzle here, but I return to Heim's analysis briefly in Sec. 6 where I provide evidence against her solution.

\section{The puzzles: ACD and Pied-Piping}

The first puzzle of concern here centers on the surprising grammaticality of (1) (capitalized material indicates stress): 
a. Sue voted for every candidate the FATHER of whom BILL had.

b. John loves every woman the MOTHER of whom BILL does.

I have checked this with at least 10 informants, all of whom have confirmed that (1) is at worst awkward (the reason for their awkwardness will, in fact, be commented on below). But under the standard view of VP Ellipsis and of Pied-Piping, sentences like (1) are quite surprising. I return to this point in greater detail later, but in a nutshell the surprise here is that the elided VP appears to be crashingly different from its antecedent. Thus one might assume that the LF (or, meaning) of the elided material in (1a) is, roughly, vote for the father of $x$. But there is no other VP with this LF or meaning.

But the plot thickens: there is an interesting asymmetry between the two VPs. To demonstrate this, we need to look at ellipsis in topicalized constructions. Thus consider (2) and (3), where no Pied-Piping is involved:

(2) a. ??Every candidate that BILL had, SUE voted for.

b. Every candidate that BILL (had) voted for, SUE did.

\section{a. ??Every woman who JOHN does, BILL loves.}

b. Every woman who JOHN loves, BILL does.

The (a) versions are slightly awkward; I assume that this is because an ellipsis site does not like to precede its antecedent. But the crucial point here is that the (b) sentences are perfectly fine. Thus the ellipsis site can be either the VP within the relative clause or the matrix VP.

But now consider the corresponding paradigm when the VP within the relative clause involves Pied-Piping. What we find here is that once again the VP within the relative clause can be elided (although there is again some awkwardness). But in this case, elision of the matrix VP leads to robust ungrammaticality (these judgments have been confirmed by about 8 informants; note too that there is no stress pattern which will improve the (b) sentences):
a. ??Every candidate the FATHER of whom BILL had, SUE voted for.
b. *Every candidate the FATHER/father of whom BILL had voted for, SUE did.
a. ??Every woman the MOTHER of whom BILL does, JOHN loves.
b. *Every woman the MOTHER/mother of whom BILL loves, JOHN does.

As noted above, the (a) sentences are perhaps questionable, but this is not surprising. In the first place, we have seen that the non-Topicalized counterparts in (1) are themselves a bit awkward. Moreover, the ellipsis site in general is not entirely happy when it precedes its antecedent (as in (2a) and (3a)). But the mystery here is that the (b) sentences are quite terrible on the intended reading given the pattern in (2) and (3) one would expect these to be much better than the (a) sentences. (There are some speakers who accept the (b) sentences, but only under the irrelevant reading in which in, e.g. (5b), John is loving the mother of the women in question. The reading of interest is the one in which John loves the women themselves, and this is impossible.) We now turn to the background pieces which, when taken together, provide an immediate solution to both puzzles. 


\section{Background}

\subsection{Rooth (1992)}

Rooth (1992) proposes that VP Ellipsis is subject to two conditions. The first is the usual identity condition: the elided VP must be identical to some other (overt) VP. Note that although this is usually phrased in terms of identity of LF (see, e.g., Sag, 1976; Williams, 1977; and much subsequent literature) one can equally well take the identity condition to be identity of meaning (Partee and Bach, 1981). Since the framework here is committed to having no level of LF, the identity condition obviously would have to be stated in terms of meanings (i.e., model-theoretic objects). While a number of interesting issues surround this debate, space precludes discussion of these; I will simply assume the identity of meaning approach in elucidating the solution.

Rooth's second condition is the focus condition, as follows: Consider an ellipsis site which I will refer to as VPEll and its antecedent, which I notate as $V_{\text {Ant }}$ Then VPEll must be contained within some constituent $\mathrm{C}_{\text {Ell }}$ and $\mathrm{VP}_{\text {Ant }}$ must be contained within some constituent $\mathrm{C}_{\mathrm{Ant}}$ such that the meaning of $\mathrm{C}_{\mathrm{Ant}}$ - or something entailed (or even implicated) by the meaning of $C_{A n t}$ - is a member of the focus value of $\mathrm{C}_{E l l}$ (i.e., the set of alternatives to the meaning of $\mathrm{C}_{E l l}$ ). (I assume that it must in fact be a proper alternative to the meaning of $\left.\mathrm{C}_{E 11}\right)$. A typical example is (6): (the underlined VP here is VPAnt):

$$
\text { [C-Ant John will leave] and [C-Ell BILL will Ø] too. }
$$

The Focus value of BILL will leave (which here is $\mathrm{C}_{\mathrm{Ell}}$ ) is a set of propositions (roughly) of the form FUT(leave'(a)) which vary on a; hence the proposition FUT(leave' $(j))$ is a member of the focus value of $\mathrm{C}_{\text {Ell }}$. Notice that Rooth's condition does not require that the meaning of $\mathrm{C}_{\text {Ant }}$ be a member of the focus value of $\mathrm{C}_{\mathrm{E} l l}-\mathrm{C}_{\text {Ant }}$ need only entail or implicate something which is a member of the focus value of $\mathrm{C}_{\mathrm{E} l l}$. Following Rooth, we refer to such cases as instances of implicational bridging. An example modified from Rooth is (7):

(7) First [C-Ant John told Mary that I would win], and then [C-Ell SUE heard that I would $\varnothing]$.

The meaning of $\mathrm{C}_{\text {Ant }}$ implicates the proposition Mary heard that I would win. Since this is an alternative to $\mathrm{C}_{\mathrm{Ell}}$ the focus condition is satisfied. (Note that since Sue is the stressed material in the second clause, it follows that $\mathrm{C}_{\mathrm{El}}$ must indeed be the constituent indicated above, since no smaller expression has a non-singleton set of alternatives.)

Before leaving this, there is one further issue which will be relevant to the exposition. The literature contains two approaches to VP Ellipsis in general. On one, the phenomenon involves deletion of the "missing" VP (cf., Ross (1967), Sag (1976), and more recent treatments such as Fox (1995)). On the second, there is no level of representation in the syntax at which the "missing" VP is present rather, the interpretation involves picking up a meaning (or, copying of the LF). (See Williams, 1977, Partee and Bach, 1981.) The second view is the one most compatible with the spirit of the theory under consideration here, as it requires no abstract levels in the syntax. I will, however, try to keep most of the remarks neutral between the two views, which will necessitate a rather informal treatment of the ellipsis phenomenon. 


\subsection{Variable-Free Semantics (Jacobson, 1992b; 1996; to appear)}

The second piece needed to crack the puzzle is the view of variable-free semantics developed in a series of papers of mine (see especially Jacobson, to appear). The basic idea here is that there are no variables and no assignment functions as part of the semantic machinery - that is, meanings are not relative to assignment functions. Although I will in fact use variables (but never unbound ones) in my representations, this is for notational convenience only - no linguistic expression has as its meaning a function from assignment functions. I do not have the space to present the full details of this theory nor its motivation; I will just sketch those parts which are crucial to the case at hand, and refer the reader to these other papers for further details. Thus consider (8); the indices here are simply used to indicate the relevant reading and are taken to have no status within the grammar:

Every man $_{\mathbf{i}}$ thinks that he $\mathrm{i}_{\mathbf{i}}$ lost.

Under the standard view, the meaning of the embedded $\mathrm{S}$ is the open proposition $\underline{\text { lost' }(\mathrm{x})}$. Put differently, he lost has as its meaning is a non-constant function from assignment functions to propositions. (In fact, all constituents have meanings which are functions from assignment functions to something else, but closed expressions are constant functions.) Under the variable-free account, the meaning of he lost is instead a function from individuals to propositions. That is, its

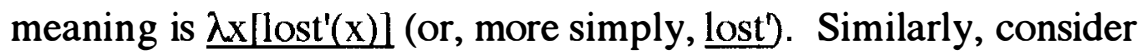

(9) Every man $_{\mathbf{i}}$ loves his $\mathrm{i}_{\mathbf{i}}$ mother.

In the standard view, his mother denotes an "open individual" - i.e., a nonconstant function from assignment functions to individuals. In the variable-free account the meaning of this constituent is instead a function from individuals to individuals. More particularly, its meaning is $\lambda \mathrm{x}[$ the-mother-of' $(\mathrm{x})]$, or, more simply, the-mother-of'. Thus in general any constituent $C$ which contains (or is) a pronoun which is unbound within $\mathrm{C}$ denotes a function from individuals to something else.

Three questions immediately arise. First, how do the pieces combine so as to give this result? Second, how does this view account for the fact that pronoun containing constituents have (essentially) the same syntactic distribution as corresponding expressions without pronouns? After all, the view here claims that a constituent with a pronoun unbound within it is not of the same semantic type as a corresponding expression with no unbound pronouns: why, then, do they have the same distribution? (Note that one of the great appeals of the standard view centers precisely on this observation. In the standard view, all expressions are functions from assignment functions to something else; the difference between an expression with no unbound pronouns and a corresponding one with unbound pronouns is not a difference of semantic type. Rather, the difference is simply that in one case we have a constant function while in the other case we have a non-constant function. Therefore, the fact that they have the same distribution comes as no surprise under the standard view.) And third, how does the pronoun eventually get "bound"?

As to the first question, I assume first that an ordinary pronoun such as he is also a function of type $<\mathrm{e}, \mathrm{e}\rangle$ - in particular, it is the identity function on individuals. Consider now (8). Here lost is listed in the lexicon with a meaning of type $<e, t>-$ how, then, can it take a pronoun (or, a constituent containing a pronoun) as subject, rather than an ordinary individual subject? The answer is that 
it undergoes the type-shif $t$ rule known in much of the Categorial Grammar literature as the "Geach rule" (and which I will notate as g), as given in (10):

Given a function $\mathrm{f}$ of type $<\mathrm{a}, \mathrm{b}>, \mathbf{g}(\mathrm{f})$ is a function of type $<<\mathrm{c}, \mathrm{a}>,<\mathrm{c}, \mathrm{b}>>$ such that $\mathbf{g}(\mathrm{f})=\lambda \mathrm{V}[\lambda \mathrm{C}[\mathrm{f}(\mathrm{V}(\mathrm{C}))]]$ (for $\mathrm{V}$ of type $<\mathrm{c}, \mathrm{a}>$ and $\mathrm{C}$ of type $\mathrm{c}$ ).

Notice that $\mathbf{g}$ is just a unary (Curry'ed) version of function composition; for two functions $\mathrm{f}$ and $\mathrm{g}, \mathbf{g}(\mathrm{f})(\mathrm{h})=\mathrm{f} \mathrm{o}$. In (8) lost undergoes the $\mathrm{g}$ rule, shifting it from type $<e, t>$ to type $<<e, e>,<e, t>>$ with meaning $\underline{\lambda f}[\lambda x[\operatorname{lost}(f(x))] l$. In other words, lost now wants a function (of type <e,e>) as subject, and it will "pass up" the argument slot of that function for binding higher up. Thus when it combines with he it takes as argument $\underline{\lambda y[y] ; ~ t h i s ~ u l t i m a t e l y ~ y i e l d s ~ l o s t ' ~ a s ~ t h e ~ m e a n i n g ~ f o r ~}$ the entire embedded sentence. The existence of the $g$ rule now also provides an immediate answer to the second question. It follows that any verb which can take an argument of type a can instead take an argument of type <e,a $>$ - i.e., an expression with an unbound pronoun within it. This is because the verb can freely type-shift by the $\mathrm{g}$ rule.

As to the binding effect, this is brought about by a second type-shift rule which in this case operates on the meaning of thinks; I have called this rule $\mathbf{z}$. Since this plays no role in the remarks in this paper I will not spell it out formally here; suffice it to say that it maps ordinary thinks' (of type $<t,<e, \downarrow\rangle$ ) into a new verb of type $<<e, \downarrow,<e, \downarrow>$, and the semantics is such that it "merges" the two eslots. In other words, like the $\mathbf{g}$ rule, $\mathbf{z}$ maps a verb into something which wants a functional object. But unlike $\mathbf{g}$, it "binds" the argument slot of that function to a higher argument slot of the verb which undergoes the shift (rather than passing the slot up for higher binding). The same remarks apply in (9). Here loves' undergoes the $\mathbf{z}$ rule, and this "binds" the pronoun-slot within the object to the subject slot.

We see, then, that the fact that expressions with pronouns have the same distribution as those without does not entail the standard view, according to which both kinds of expressions have the same type of meanings (where both are functions whose domain is the set of assignment functions). The distributional regularity also follows if the grammar contains the $\mathrm{g}$ rule. But does the introduction of the g rule represent a large "cost"? In Jacobson (to appear) I argue that it does not; here we will further see that this rule has a striking payoff.

\subsection{ACD as TVP Ellipsis (Cormack, 1985; Jacobson, 1992a, 1992b)}

The third background piece concerns the analysis of ACD proposed originally in Cormack (1985) and further developed in Jacobson (1992a, 1992b). Consider a typical ACD case (from Bouton, 1970):

John read every book which Bill did.

The interest in ACD for the general program here is that the existence of (11) is usually taken to be evidence both for a level of LF at which the object is "raised" out of its surface position, and for the existence of a level of representation at which there is a variable in object position. Thus the "received" position on ACD goes like this. (1) Relative clause meanings are put together in such a way that the material following which must (at some point in its life) denote an open proposition (open on some variable over individuals; this variable eventually gets closed by $\lambda$ - 
abstraction). From this it follows that: (2) The elided or missing material here must be a VP meaning (or, LF), where the missing VP will contain an individual variable in object position. (3) It thus follows in turn that the antecedent must be some expression which has a VP-type of meaning (or, LF). (4) But on the surface, there is no full VP which can supply the missing meaning (or, LF). The only full VP itself contains the ellipsis site. Hence if we used this VP to determine the meaning of and/or LF of the "missing" material, we arrive at a paradox. (5) Thus, in order to avoid the antecedent containment paradox, we need a level of LF at which the object is pulled out and where there is a variable in its place. In other words, the LF for (11) is (12); from here we can find the antecedent read $\mathrm{x}$ which can serve as the antecedent for the ellipsis:

every x: [book, $\mathrm{x} \&$ John read $\mathrm{x}$ ] [Bill did read $x$ ]

Cormack (1985), however, shows that once one rejects the original assumption in (1), there is no antecedent containment paradox here: the "missing" material can simply be a 2-place relation rather than a VP meaning. Thus the antecedent can simply be a transitive verb phrase (in this case, the simple transitive verb read). To elucidate, we will consider first the semantic composition of a nonellipsis case like (13) under a Categorial Grammar account of the semantics of relative clauses:

John read every book which Bill read.

Actually, there is more than one (closely related) approaches to extraction within the general CG program (see, e.g., Steedman, 1987; Jacobson, 1989; Oehrle, 1990); for expository ease I will adopt the approach of Steedman (1987), although similar remarks will for the other approaches. Thus assume that the way the meaning is put together here is that the 2-place relation read' simply functioncomposes with the (type-lifted) meaning of Bill, as follows:

$$
\left.\lambda P[P(b)] \text { o read' }=\lambda x\left[\operatorname{read}^{\prime}(x)(b)\right]\right]
$$

Notice, incidentally, that in the "standard" account this also is the eventual meaning of Bill read; the usual view is that we first compose the meaning $\operatorname{read}^{\prime}(x)(b)$ which contains an open variable, but this variable is then closed off by $\lambda$ abstraction. In the CG account sketched above the semantics directly yields the property rather than invoking the process of $\lambda$-abstraction (note that $\lambda$-abstraction itself is a type-shift rule, which in this case maps an open proposition into a closed property). Since there is no stage in the semantic composition in which there is an open variable, this is a variable-free analysis of the semantics of extraction. There is no expression whose meaning (at any stage in the semantic composition) contains an open variable and thus must be seen as a function from assignment functions; there is also no need for a trace in the syntax to "correspond" to a variable in the semantics. What happens in the rest of the semantic composition depends on the precise analysis of the relative pronoun which. For now let us simply assume that which Bill read has the same meaning as Bill read (thus, it is the property $\left.\lambda x\left[\operatorname{read}^{\prime}(\mathrm{x})(\mathrm{b})\right]\right)$, and that this then intersects with the meaning of the head noun. (See Sec. 3.4 for discussion of the meaning of which.)

The idea, then, is that in an ACD case, exactly the same thing happens. In (11), the "missing" meaning is simply the two-place relation read'. This function composes with did', and did'(read') in turn function composes with the (type- 
lifted) meaning of the subject. The meaning of the relative clause is thus the

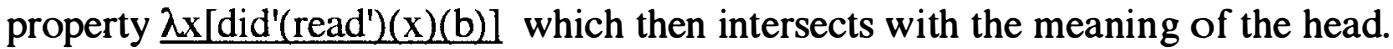
The crucial point to notice about this analysis is that here the antecedent of the ellipsis site is smaller than in the standard view - it is only a Transitive Verb (Phrase) and not a full VP. It is precisely this fact - combined with the existence of the $\mathbf{g}$ rule - which will be key in solving the puzzle of the grammaticality of (1).

But before continuing, a few points should be clarified. First, it is wellknown that $\mathrm{ACD}$ can involve an ellipsis which is a much bigger chunk than a simple transitive verb, as in (15) (the italicized material indicates the intended reading of the ellipsis site):

John thought that Mary read everything that Bill (also) did $\varnothing(\varnothing=$ think that Mary read).

Moreover (contra Baltin, 1987), the antecedent material need not even be continuous, as in (16) (Larson and May (1990)):

John put every book that Bill also did $\varnothing$ on the shelf $(\varnothing=$ put on the shelf $)$.

(15) is discussed in detail in Cormack (1985), and (16) in Jacobson (1992a). As to (15), there is no problem here if we assume that the phrase thought that Mary read in the antecedent position can compose together to give a complex transitive verb (phrase), which supplies the missing 2-place relation. Moreover, both Cormack and Jacobson show that this general view also accounts for the observations of Sag (1976) regarding the interaction of de re and de dicto readings in ACD. In Jacobson (1992a) I show there that (16) is unproblematic in a view of the syntactic combinatorics which includes a Wrap operation; put on the shelf is a single expression (whose meaning is a 2-place relation) into which the object is Wrapped. Additional evidence for the Wrap plus TVP analysis is detailed in Jacobson (1992a).

A more serious worry is one pointed out to me by a SALT reviewer: the TVP Ellipsis analysis must ultimately explain why this phenomena does not have the same distribution as Pseudo-Gapping, which is also a case of TVP Ellipsis. Thus, for example, compare (17a) and (17b):

a. John thought that Mary read everything that Bill (also) did $\varnothing(\varnothing=$ think that Mary read).

b. * John thought that Mary read Crime and Punishment, and Bill did $\varnothing$ The Brothers Karamazov ( $\varnothing=$ think that Mary read).

Roughly, pseudo-gapping involves only the elision of a simple transitive verb (or a verb plus preposition) - not larger phrasal material. I do not have a full story to tell here, but I suspect that the account of this difference will lie in the syntax of the two constructions. That is, (unlike Lappin, 1996) I am not claiming that ACD reduces to pseudo-gapping. While both involve the supplying of a missing 2-place relation in the semantics, what licenses the missing material in the syntax would differ in the two cases. Admittedly this answer is preliminary: the TVP Ellipsis analysis does ultimately need to spell out just what licenses the missing material in each case, and why the syntax is different. I leave this matter open here.

Finally, I have been rather vague about the notion of "picking up a meaning" and just how and where in the semantic composition this happens. I will, in fact, continue to be vague on just this point, simply because I do not have space for a sensible discussion. But note that the need to make this explicit is independent 
of the TVP Ellipsis analysis - the same question arises with respect to VP Ellipsis as well. (Note too that this question will be moot if VP/TVP Ellipsis turns out to be deletion.) Incidentally, while I assume that the semantics of ACD is TVP Ellipsis, I will continue to assume that this - like ordinary VP Ellipsis - is subject to both the identity condition and to Rooth's focus condition. The story to be told in Sec. 4 does in fact show that the focus condition must be satisfied here.

\subsection{Pied-Piping in a variable-free (trace-free, reconstruction-free) account}

Before returning to the puzzles in Sec. 2, there is one more background piece to develop: the analysis of Pied-Piping under the view of semantics which embraces strict surface compositionality. What is striking here is that the semantics of PiedPiping is automatic given the variable-free program in Sec. 3.2: nothing extra is needed to handle the semantics without recourse to reconstruction and/or LF. Thus consider a run-of-the-mill Pied-Piping case like (18):

$$
\text { every candidate the father of whom Bill voted for }
$$

In order to provide an interpretation for (18), there is no need to put the Pied-Piped material into the position of the gap. Rather, we can take the missing object argument to be a function of type <e,e> (see also Sharvit, 1997 for a similar analysis of Pied-Piping in questions). The interesting point to notice here is that the possibility that the gap is of type $<e, e>$ is an automatic consequence of the existence of the $\mathbf{g}$ rule. Assume, then, that the meaning of Bill voted for is put together, as discussed earlier, by function composition of vote-for' with the (type-lifted) meaning of Bill. This means that this material combines to give the property

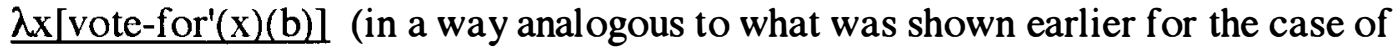
Bill read). But now recall that anything can undergo the $g$ rule in such a way that instead of wanting as argument something of type a, it will want an argument of type $<\mathrm{e}, \mathrm{a}\rangle$. In this case the meaning of Bill voted for wants an argument of type $\mathrm{e}$; if it undergoes the $\mathrm{g}$ rule it will then want an argument of type $<e, e>$. In other words, the derivation will be as follows:

$$
\begin{aligned}
& g(\lambda x[\text { voted-for' }(x)(b)])=\lambda f\left[\lambda y\left[\lambda x\left[\text { voted }-f^{\prime}{ }^{\prime}(x)(b)\right](f(y))\right]\right]= \\
& \lambda f[\lambda y[\text { voted-for'(f(y))(b)] }
\end{aligned}
$$

This, then is of type $<<e, e>,<e, t>>$ - it wants a function of type $<e, e>$ as argument, and it will return an ordinary property.

Consider now the father of whom. Suppose that relative pronouns are like ordinary pronouns, and thus denote the identity function on individuals. In that case, the father of whom will have as its meaning the-father-of' - exactly like his father has this as its meaning. In other words, if we make the (rather reasonable) assumption that relative pronouns have the same kind of meaning as ordinary pronouns, it follows immediately that the father of whom is of type $<\mathrm{e}, \mathrm{e}\rangle$, and thus has the right sort of meaning to be argument of $\mathbf{g}$ (Bill' o voted-for'). The result, when this occurs as argument of Bill voted for is as shown in (20)

(20) the father of whom Bill voted for; $\lambda f\left[\lambda y\left[\operatorname{voted}_{-} \operatorname{for}^{\prime}(f(y))(b)\right]\right]($ the-father-

$$
\text { of } \left.^{\prime}\right)=\lambda y[\text { voted-for'(the-father-of'(y))(b)]] }
$$


(This is similar to the analysis of Pied-Piping in Sharvit (1997), except that hers is not implemented in a variable-free semantics, and thus requires some extra apparatus. For example, some step is needed to $\lambda$-abstract over the open variable in the meaning of the father of whom in order to turn it into a closed function of type $<e, e>$.) Incidentally, if the relative clause is introduced by an ordinary relative pronoun (as in every candidate who Bill voted for) the derivation is probably exactly parallel. Since the relative pronoun is again of type $<\mathrm{e}, \mathrm{e}>$ we can assume that here too the $\mathbf{g}$ rule applies to allow for this to be argument of Bill voted for . In this case the functional argument (the relative pronoun) is simply the identity function.

\section{Solving the puzzles}

We are now in a position to crack the puzzles in Sec. 2. First I will show in this section that all of the pieces fall into place once we assume the three background pieces above (Rooth's analysis; the $g$ rule; and the ACD as TVP Ellipsis analysis). I will then show in Sec. 5 that the story does not translate straightforwardly into the "standard" view (according to which there is a level of LF, ACD is treated as VP Ellipsis where VPAnt and VPEll are both VPs which contain unbound variables in object position, and where - consequently - the semantics makes crucial use of variables).

Our first concern will be with the good cases:

Sue voted for every candidate the FATHER of whom BILL had.

??Every candidate the FATHER of whom BILL had, SUE voted for.

Let us first consider whether there is a way to satisfy the identity condition. The answer is yes: this is perfectly trivial. The analysis of (1a) is essentially just like that of (18), where the missing 2-place relation here is simply the meaning of the TVP voted for. This function-composes with had' and had'o voted-for' then composes with the (type-lifted) meaning of Bill, to give the property

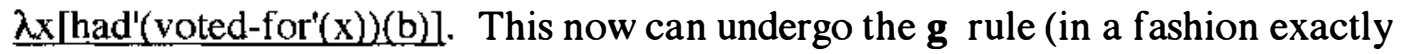
analogous to what we saw for (18)). This will map the above property into the meaning: $\left.\left.\quad \underline{\lambda f}\left[\lambda \mathrm{y}[\lambda \mathrm{x}] \mathrm{had}^{\prime}\left(\operatorname{voted}_{-} \operatorname{Cor}^{\prime}(\mathrm{x})\right)(\mathrm{b})\right](\mathrm{f}(\mathrm{y}))\right]\right]$ which is equivalent to $\lambda f\left[\lambda y\left[h a d '\left(\operatorname{voted}-f_{0}(f(y))(b)\right] l\right.\right.$. The meaning of the father of whom is again the-father-of'; when this occurs as argument of the meaning of BILL had we get the property $\lambda y[$ had'(voted-for'(the-father-of'(y)) )(b)], and this then intersects with candidate'. Thus the identity condition has no difficulty in being satisf ied because the antecedent need only be a missing 2-place relation (as expected in the TVP ellipsis analysis). The fact that the gap can be of type $<$ e,e $>$ (and hence the fact that BILL had can co-occur with something like the FATHER of whom) is simply a consequence of the fact that any expression - including this one - can undergo the $\mathbf{g}$ rule. (And note that if the remarks following the discussion of (20) are correct, the $\mathrm{g}$ rule applies even in the ordinary case of ACD with an ordinary (non-Pied-Piping) relative pronoun.)

Our next question is whether Rooth's focus condition is also met here. Again the answer is yes, although this is not obvious at first glance. Notice first that these are good only with the stress as indicated above. In (1a) for example, both FATHER and BILL require stress (Sue can also be stressed). In (4a) again there must at least be stress on these two expressions. To simplify the exposition, 
we will begin with a consideration of the topicalized version in (4a). Suppose that we analyze $C_{E l l}$ as the expression the FATHER of whom BILL had. Its meaning (once we supply the missing TVP meaning) is $\underline{\lambda y}$ [had'(voted-for'(the-fatherof' $(\mathrm{v}))(\mathrm{b}) \mathrm{l}$; for simplicity I will henceforth ignore had', and so will rewrite this meaning as $\underline{\lambda y}[$ voted-for'(the-father-of-(y))(b)]. Now since BILL and FATHER are both stressed, this means that the focus value of this expression (i.e., the set of alternatives) are alternative properties of the form $\lambda y[$ voted-for' $(f(y))(a)]$ which vary on the value of $\underline{f}$ and of $\underline{a}$. In other words, the focus value is the set of properties (represented in a more prose-like way): $\{\lambda y$ [Sue voted for the mother of $y], \lambda y$ [Sue voted for the sister of $y l, \lambda y$ Mary voted for the mother of $y l . .$.$\} .$ Consider now $\mathrm{C}_{\text {Ant }}$, which can be $S U E$ voted for. Its meaning is simply the property $\lambda y[$ Sue voted for $(y)]$ - as there is no function of type <e,e> here this would seem not to "count" as an alternative to the meaning of $\mathrm{C}_{\text {Ell. }}$. But recall that the alternative can be something entailed or even implicated by the meaning of $\mathrm{C}_{\text {Ant }}$ - and from the property $\underline{\lambda y}[$ Sue voted for $(y)]$ we can surely derive the property $\underline{\lambda y[S u e ~ v o t e d ~ f o r ~(i d(y)) l, ~ f o r ~ i d ~ t h e ~ i d e n t i t y ~ f u n c t i o n ~(o n ~ i n d i v i d u a l s) . ~ I n ~ o t h e r ~}$ words, we can "access" an alternative to the-father-of' function, since the identity function should be available. There are two (related) points which should be clarified here. First, if we are claiming that the "accessing" of the identity function is done by implicational bridging, then we need some way to extend the notion of entailment to properties (since the meaning of $\mathrm{C}_{\mathrm{Ell}}$ is a property), but such an extension would be straightforward. But second, it is not actually clear to me that we need to invoke implicational bridging here. After all, $\underline{\lambda y}[$ Sue voted for (id(y))] is equivalent to $\underline{\lambda y[S u e ~ v o t e d ~ f o r}(y)]$ and thus the actual meaning of $C_{E l l}$ is indeed a member of the focus value of $\mathrm{C}_{\text {Ant }}$.

Nonetheless, one might have the feeling that invoking the identity function as the alternative to the-father-of' function is a bit of a cheat. But in fact there is evidence that this move is entirely legitimate, for there are other cases where the identity function is treated as an honest-to-goodness alternative to "normal" functions of type $<e, e>$. Consider, for example, the contrast between (22) and (23):

John loves only Bill's MOTHER. Not Bill's father, not Bill's sister, not Bill's aunt, - not even Bill (himself).

??John loves only Bill's MOTHER. Not Bill's father, not Bill's sister, not Bill's aunt, - not even Tom.

(23) is rather strange. To the extent that it is possible, this is probably because stress on MOTHER can be an instance of broad focus, where the entire NP Bill's MOTHER is the focused constituent. (Hence the set of alternatives is a set of ordinary individuals - including individuals like Bill's father, Bill's sister, and also Tom.) Note, though, that in (23), Tom is preceded with a list of functions (Bill's father, Bill's sister, Bill's aunt); this is intended to stack the deck towards the narrow focus interpretation. In other words, this strategy favors an interpretation in which it is only MOTHER which is focused - hence the set of alternatives to Bill's MOTHER are alternatives of the form $\underline{\mathrm{f}(\mathrm{Bill})}$ ) which vary on the value of $\underline{\mathrm{f}}$. Given this, there is a feeling of surprise when we encounter Tom - for this denotes just an ordinary individual, and not some function of type $<\mathrm{e}, \mathrm{e}>$ applied to Bill'. 
Now compare this to (22) - here there is no similar feeling of surprise. Yet Bill like Tom - also denotes just an ordinary individual, so why should there be no "surprise" here? Presumably because Bill can mean (or allow us to access) id(Bill') - where the identity function is just the sort of function which counts as being an alternative to the mother-function. (Note too that (22) is especially good if Bill is followed by himself - himself seems here to be nothing more than an overt item meaning the identity function.) Thus it appears that the identity function does, indeed, "count" as a wholesome alternative to more run-of-the-mill functions of type $<e, e>$. Incidentally, the fact that the identity function in (4a) has no overt manif estation and so must be "accessed" by some sort of inference process may well explain why these types of cases involving ACD and Pied-Piping do have a slight awkwardness to them.

A similar story can be told for the non-Topicalization case as in (1a), although this does require some comment. Again I assume that $\mathrm{C}_{\text {Ell }}$ is the FATHER of whom Bill had - and so its focus value is a set of properties of the

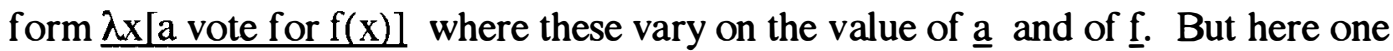
might wonder what counts as $\mathrm{C}_{\mathrm{Ant}}$ - since here it is not obvious that Sue vote for is a surface expression. I will assume here the general line of, e.g., Steedman (1987), by which even in the case where there is no Topicalization, this material can indeed be composed in the syntax to give such an expression which then combines with its object. (Alternatively, it may be that the property $\underline{\lambda x[S u e ~ v o t e ~ f o r ~} \mathrm{id}(\mathrm{x})]$ is accessed by some kind of implicational bridging, but this line would require a fuller account of just how and where implicational bridging can take place.) Incidentally, the same question arises in this account with respect to a simple case like (24):

$$
\text { John read every book which BILL did. }
$$

If $\mathrm{C}_{\mathrm{Ell}}$ is (which) BILL did, then its meaning (once the missing 2-place relation is

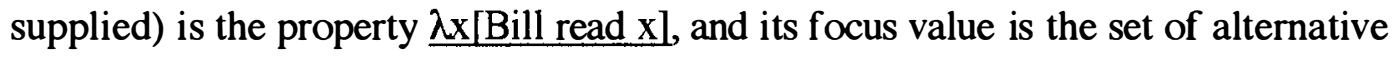

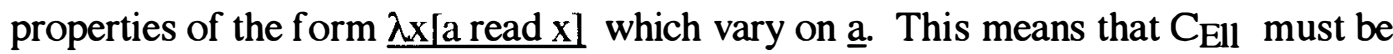
John read, and so we will again assume that this material can be combined in the syntax by function composition to form a constituent. (Again the same alternative

presents itself: it could be that the property $\underline{\lambda x[\text { John read } \mathrm{x}]}$ is arrived at by some kind of "implicational bridging", but again this would require fleshing out this notion in more detail.)

Let us now turn to the second puzzle: the asymmetry between the two VPs. In particular, why are (4b) and (5b) bad? Take (4b):

*Every candidate the father of whom BILL had voted for, SUE did.

Here $C_{E l l}$ would have to be $S U E$ did $\varnothing$. Consider the question of whether the father of whom BILL had voted for is possible as $\mathrm{C}_{\text {Ant }}$. Notice that the focus value of $S U E$ did $\varnothing$ (once we supply the missing 2-place relation) are alternative

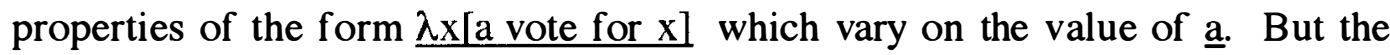
meaning of the father of whom BILL had voted for is the property $\underline{\lambda x \text { [Bill voted }}$ for the father of $x]$ - and so it is not an alternative to $C_{E l l}$. But, one might wonder, why could we not play the same "trick" that we played earlier? That is, since the meaning of $S U E$ did $\emptyset$ is the property $\underline{\lambda x[S u e ~ v o t e d ~ f o r ~} \mathrm{x}]$, and since that is equivalent to $\underline{\lambda x[S u e ~ v o t e d ~ f o r ~} \mathrm{id}(\mathrm{x})]$, could we not set things up in such a way that 
the focus value of $S U E$ did $\emptyset \quad$ is a set of alternative properties of the form $\underline{\lambda \times \text { [a }}$ vote for $f(x)]$ where these alternatives vary on the values for $\underline{f}$ and for $\underline{a}$ ? If this were possible, then indeed the property $\lambda x$ [Bill voted for the father of $x$ ] would be a legitimate alternative, and so the focus condition should be met.

But indeed this is not possible, for there is no way to give focus to the identity function in the meaning of $S U E$ did $\varnothing$, since there it has no lexical realization here and therefore nothing which can be stressed. (For related observations on a somewhat different case, see Heim, 1997.) It is this fact, then, which is exactly the source of the asymmetry between the two constituents. The expression the FATHER of whom BILL did $\varnothing$ can be $\mathrm{C}_{\mathrm{E}}$ all and can find $S U E$ voted for as its $\mathrm{C}_{\text {Ant }}$ because here father is overt and hence capable of receiving

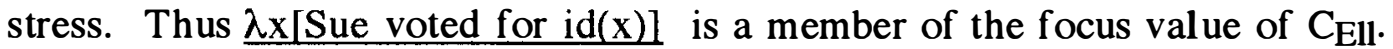
When the situation is reversed there is no way to set up a focus value for $S U E$ did $\varnothing$ in such a way that it is looking for alternatives to the identity function, because this cannot be stressed. This asymmetry between the two expressions thus provides very interesting confirmation for Rooth's condition (again, see also Heim, 1997 for related discussion).

The reader may have noticed one loose end. Why can't $C_{A n t}$ in $(4 b)$ be the smaller expression BILL (had) voted for? Of course, once the meaning of this expression undergoes the $\mathbf{g}$ rule the semantic types will not be right, as its meaning is $\lambda f[\lambda x[B$ ill vote for $f(x)]]$ and so it is not of type $<e, \downarrow$ and hence not a possible alternative to the property $\underline{\lambda x[S u c}$ vote for $\mathrm{x}]$. But this fact does not answer the above question, since we have claimed that the semantic composition can proceed in such a way that there is a stage in the composition at which Bill (had) voted for has as its meaning simply the property $\underline{\lambda x[B i l l ~ v o t e ~ f o r ~} \mathrm{x}]$ (where this property then is mapped into the above meaning by the application of the $g$ rule). This, then, should count as an alternative to the property of being voted for by Sue, and hence Bill (had) voted for should be possible as $C_{\text {Ant }}$. I do not, in fact, have a full answer to this - but this turns out (given certain assumptions) to just be a special case of the Kennedy puzzle to which I return in Sec. 6. I assume that whatever explains that puzzle will also explain the fact here.

\section{Translating this into the "standard" view}

The above story made use of the analysis of ACD as TVP Ellipsis, and also made use of a theory with the $\mathrm{g}$ rule - which in turn is motivated by the variable-free semantics. Thus if the account of these facts does require this apparatus, we see that this domain provides independent support both for the $\mathrm{g}$ rule and for the TVP Ellipsis analysis - and hence independent support for the more general program of variable-free (and LF-free) semantics. A crucial question, then, is whether or not the story can be translated into the standard view. (By the "standard" view I mean

one in which ACD is VP Ellipsis, which in turn entails that the object is raised at LF and leaves a variable in its place, and this in turn entails a theory with crucial use of variables.)

The answer is that it cannot - at least not in any straightforward way. As it turns out, the part of the story which relied on the focus condition can, perhaps, be restated in terms of the standard view. But the problem has to do with the identity condition: it is not clear under the standard view just how this can be met. Thus consider again the original puzzle, which centered on the grammaticality of (1a): 
(1a) Sue voted for every candidate the FATHER of whom BILL had.

Consider first a theory of the semantics of Pied-Piping in which the Pied-Piped material is "reconstructed" into the position of the gap. In that case, then, the LF for the elided VP would be voted for the father of $x$, while the LF for the antecedent VP is voted for $x$. Needless to say, these two VPs are crashingly different - it is difficult to imagine any non ad-hoc definition of "identity" which would encompass this case. (One might object that it makes little sense to say that the elided VP is voted for the father of $x$, since part of this material (the father of $x$ ) is overt on the surface. This is true - but it just points to a more fundamental problem. The standard view crucially says that the ellipsis site is a full VP - and so it really is not clear how to think about this case sensibly under a reconstruction analysis of Pied-Piping combined with the standard view of VP Ellipsis.)

There is a second tack which one could try. Even under the standard view, reconstruction is not necessary for Pied-Piping (see Sharvit, 1997). Thus assume that the gap is given a complex translation, where it corresponds to a variable over functions of type $<e, e>$ applied to an individual variable. (This is analogous to the analysis of functional gaps in Groenendijk and Stokhof (1983) and Engdahl (1986), except that here the variable which is the argument of the function will not be bound by the subject.) Roughly, then, the LF for (1a) would be (25):

for every $\mathrm{x}$ : $\mathrm{x}$ a candidate \& [for the $\mathrm{f}: \mathrm{f}$ is $\lambda \mathrm{y}[$ the-father-of' $(\mathrm{y})] \&$ Bill had voted $f o r f(x)$ ] Sue voted for $\mathrm{x}$

(The ellipsis site is given in italics, and the antecedent VP is underlined.) But this is of no help - the LFs are still not identical, since $f(x)$ is not the same as $\underline{x}$. Notice that the difference here does not reduce to other kinds of non-identity which have been proposed under the standard analysis. Thus some authors have suggested that the identity condition must be allowed to ignore differences in variable names (see, e.g., Sag, 1976), and others have argued that the identity condition must be allowed to ignore certain (essentially syntactic) features on the variables and/or traces (see, e.g., Fiengo and May, 1995). But here there is a totally different structure for the two objects; one is $\underline{\mathbf{x}}$ and the other is $\underline{f}(\mathbf{x})$. (Incidentally, it is worth noting that allowing the identity condition to ignore differences in variable names is itself an artifact of having variables - the variable-free semantics needs no such exception to the identity condition.)

A final tack which one might try is one suggested to me by a SALT referee. This is to look at a "pre-reconstruction" representation of the elided VP: thus we can take its representation to be voted for $x$, while the antecedent VP (after QR) has the same LF. But it is not clear what it means to call vote for $x$ the LF of the ellipsis site. In order to provide an interpretation for this material, one either needs a functional gap (as in (25)) or a reconstruction structure. This would seem to involve positing some new notion or level of LF which has no motivation save to provide a solution to this problem.

Thus the key difference between this standard view and the variable-free view has to do with the "size" of the ellipsis site. Under the TVP Ellipsis analysis, the elided material is nothing more than the 2-place relation vote-for'. The meaning of the entire expression Bill did (vote for) is what undergoes the $g$ rule, making the "gap" a functional one - but this in no way interferes with the ability of the ellipsis site to find an identical antecedent. 


\section{Kennedy's Case: Evidence for Variables?}

There is, however, a notable problem concerning ACD which remains unsolved in the present account. This problem is due to Kennedy (1994), and concerns the ungrammaticality of sentences like (26);

a. *John read every book which was reviewed in a magazine which BILL did.

b. *Every book which was reviewed in a magazine which BILL did, John read.

(I have modified Kennedy's original example to avoid some potential but irrelevant complications due to the possibility of inverse scope readings in his examples. Also, both Kennedy (1994) and Heim (1997) discuss a broader range of cases which I will not have space to consider here.) The reason why this problem is significant for the account here is that Heim (1997) proposes a solution to this puzzle which makes quite crucial use of variables - in fact, it makes a more robust use of variables than in the standard account. While I have no alternative solution to offer, I will show here that Heim's account is problematic in three respects.

Heim's analysis rests on a group of premises which, taken together, suggests that many linguistic expressions standardly taken to denote properties instead have meanings which are open propositions; she dubs this the "Formulas Hypothesis". Consider an ordinary sentence containing a relative clause, as in (27):

Every man who Mary likes left.

The Formulas Hypothesis claims that there is no stage in the semantic composition at which (who) Mary likes denotes the property $\underline{\lambda x[l i k e s}(\mathrm{x})(\mathrm{m})]$. Rather, its meaning is the open proposition likes' $(\mathrm{x})(\mathrm{m})$. Note that the standard account also claims that there is a stage in the semantic composition at which Mary likes (and perhaps who Mary likes) has this meaning, but under this account $\underline{\mathrm{x}}$ is ultimately $\lambda$-abstracted over to form a property which intersects with the head. But under the Formulas Hypothesis, no $\lambda$-abstraction ever takes place here. Obviously, then, the meaning of who Mary likes cannot intersect with the meaning of man if the latter has a meaning of type $<e, \downarrow$. Thus in Heim's account man also denotes an open proposition $\operatorname{man}^{\prime}(x)$. In other words, both the head and the relative clause denote sets of assignment functions (rather than sets of individuals), and their meanings are combined by intersection of the assignment function sets. Finally, consider how the entire sentence is put together. In the account in Heim (1997), the determiner takes both arguments at the same time (we return to this point momentarily); the LF for (27) is (28), and the rule for interpreting this is (29) (I have slightly altered Heim's formulation of (29), but the result is equivalent):

every $_{x}$ [man, $x$ \& Mary likes $\left.x\right]$ [x left]

$\left[\left[\right.\right.$ every $\left.\left._{\mathrm{x}}[\Phi][\Psi]\right]\right] \mathrm{g}=1$ iff for all assignment functions $\mathrm{g}^{\prime}$ exactly like $\mathrm{g}$ except for the value assigned to $x,\left[\left[\Phi^{\prime}\right]\right]^{g^{\prime}--->}\left[\left[\Psi^{\prime}\right]\right] g^{\prime}$.

To see how this supplies a solution to the Kennedy puzzle, consider (26). Under Heim's analysis, this has the LF in (30) (the italicized material is the ellipsis site, and the underlined material is its antecedent): 


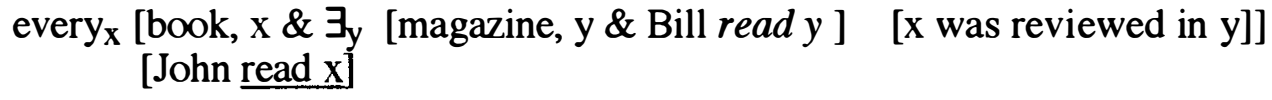

The identity condition is met here (Heim's account is forced to allow the identity condition to ignore differences in variable names). The focus condition, however is not. Suppose that $C_{E l l}$ is BILL did (read y). The set of alternatives to this open proposition are a set of propositions of the form a read $y$, which vary on a. Thus John read $x$ cannot qualif $y$ as $C_{A n t}$ - because its meaning is the proposition John read $\mathrm{x}$, not John read y. The key is that here Bill read $y$ and John read $x$ are both open sentences - rather than properties - and they are open on different variables. Notice that in the variable-free view, the meaning of the expression BILL did (once the missing 2-place relation is supplied) is the property $\underline{\lambda x[B i l l ~ r e a d ~} x]$. The set of

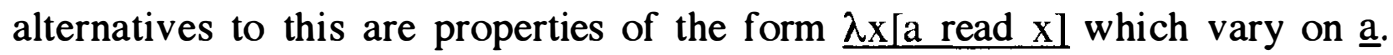
Moreover, we need to assume that John read can compose to give the property $\underline{\lambda x[J o h n ~ r e a d ~} x]$ (or that this property can be accessed in some way), and so this will in fact be an alternative to the meaning of BILL did and so the focus condition is met. The ungrammaticality of (26) thus remains a mystery. The standard view fares no better: although BILL did (read) is not born with the property meaning, it type-shifts from the open propositional meaning into the property meaning. Similarly, under the standard view the object of the matrix $S$ is raised at LF, and so there is an LF expression John read X. But the usual view of what it means for this to combine with the "raised" object (every book which was reviewed in a magazine which Bill did (read)) is that $\underline{\mathrm{x}}$ in John read $\mathrm{x}$ is $\lambda$-abstracted over, and the resulting property is taken as argument of the generalized quantifier. Thus here too we have a stage in the semantic composition where the two relevant expressions denote properties, and so one should count as an alternative to the other.

Intriguing though Heim's solution is, it nonetheless has two suspicious aspects to it and, more seriously, makes incorrect empirical predictions. First, as Heim notes, it works only if one also adopts a condition against "accidental coindexing" (one could formulate this condition in various ways; perhaps the simplest is that no two determiners in an LF be co-indexed). The most vivid illustration of why such a condition is necessary comes from a case like (31) - some principle is needed to keep it from having the LF in (32). (This is not actually the sort of case which Heim used to motivate this condition; but the particular cases she considered could be ruled out in other ways.)

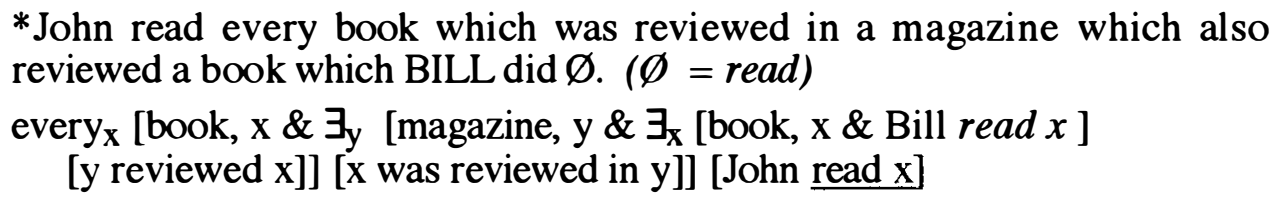

Such an LF would satisfy the focus condition, since John read $x$ is a member of the focus value of BILL read $x$. One thus needs to rule this out by a condition which ensures that the two relevant variables have different names, since they are ultimately bound by different determiners. Notice that while such a condition can certainly be formulated, it does point to the fact that Kennedy's puzzle is actually not automatically solved under the Formulas Hypothesis. It is not enough to posit that relative clauses always denote open propositions: this must be supplemented by an additional condition which would have to be formulated as a global condition which inspects the entire LF to ban reuse of the same variable name. 
The second suspicious aspect about this kind of solution concerns the fact that the identity condition must be allowed to ignore differences in variable names, while the focus condition cannot. But why should this be? Of course the focus condition is sensitive to semantic objects - and an open proposition like John read $\mathrm{x}$ is indeed a different semantic object from an open proposition like John read y. One might therefore argue that it is of course automatic that the focus condition will be sensitive to the variable names. But the same reasoning will lead us to expect the identity condition to care about variable names, too. Suppose (as is standard) that the level relevant for identity is LF. Then it is true that identity is syntactic (i.e., reprsentational) identity, rather than semantic identity - but why does this matter? After all, read $x$ and read $y$ are different syntactic objects (i.e., different representations) every bit as much as they are different semantic objects and so the fact that variable names don't "count" for identity here remains mysterious. As noted above, under the variable-free semantics the entire issue of non-identical variable-names becomes moot: there are no variables and hence no variable names, and so the identity condition (which would be semantic identity here) need not "ignore" this difference.

But most seriously, Heim's analysis has empirical problems. Consider (33), which is impeccable:

Every woman who John loves spoke to every woman who BILL does.

As the interested reader can verify, here $\mathrm{C}_{\text {Ant }}$ and $\mathrm{C}_{\mathrm{Ell}}$ could be nothing larger nor nothing smaller than the two NPs underlined above. But will these do? Under the Formula's Hypothesis they in fact will not - and so this incorrectly rules out cases like this. Note first that under Heim's actual formulation of the semantics, these constituents have no meaning at all - because the Determiners take both arguments at the same time. But this of course is perfectly trivial to remedy: we simply Curry the determiners such that they take their arguments one at a time. I will not spell out the full meaning of every, but its meaning would be such an NP of the form every $\Phi$ will have the semantics in (34):

Let $\mathrm{G}$ be a variable of type $<\mathrm{g}, \triangleright$ (i.e., a set of assignment functions)

Let $\Phi^{\prime}$ be the semantic value of $\Phi$ - hence $\Phi^{\prime}$ is of type $<\mathrm{g}, \downarrow$

Then: the meaning of every $y_{x} \Phi=$

$\lambda G\left[\lambda g\left[\forall g^{\prime}\right.\right.$ exactly like $g$ except for the value assigned to $x$,

$$
\left.\left.\left[\Phi^{\prime}\left(g^{\prime}\right)-->\mathrm{G}\left(\mathrm{g}^{\prime}\right)\right]\right]\right]
$$

(There are other slightly different possible formulations, but all will have the same effect.) Thus in a case like every man left, every (man ( $\mathrm{x}$ ) ] will first combine to give the meaning as given above, and this will take as argument $\underline{x}$ left.

But given this semantics (or any other reasonable semantics which I have been able to think of), the meaning of the expression every $\mathrm{x}$-woman, $\mathrm{x}$ \& John loves $x$ ] is not a member of the focus value of the expression every ${ }_{y}$ [woman( $y$ ) and Bill does (love y)]. Although it is not so obvious just from the representations, the point is that these two are also both actually open expressions - and they are open on different variables. The simplest way to convince oneself of this is to note that the following two expressions are not the same semantic objects every [man, $x]$ and cvery $y_{y}[$ man, $y]$. Both expressions are functions from sets of assignment functions, but the values that they assign to any set of assignment functions depends on the index on the determiner: 
Note that neither the standard theory nor the variable-free theory have any difficulty in predicting the grammaticality of (33). In either of these theories, the meanings of the underlined constituents are closed expressions - they are sets of sets of individuals, and the meaning of the subject generalized quantifier will be a member of the focus value of the object generalized quantifier. But of course the variable-free and standard theories in turn have no account for the Kennedy cases in (26), since here too we are dealing with closed expressions. I conclude, then, that the contrast between (26) and (33) remains a puzzle - regardless of the status of variables. In other words, the full range of facts here is not compatible with the Formulas Hypothesis - and thus does not constitute evidence for a theory making crucial use of variables.

\section{Endnotes}

* For helpful discussion of the material here, I would like to thank Irene Heim, Manfred Krifka, Fred Landman, and Mats Rooth. The research was conducted while I was a fellow at the Hebrew University of Jerusalem Institute for Advanced Studies, whose support is gratefully acknowledged.

\section{References}

Baltin, M., 1987. "Do antecedent-contained deletions exist?', Linguistic Inquiry 18.

Bouton, L, 1970. "Antecedent contained pro-forms", in M. Campbell et al., (eds.), Proceedings of the 6th Regional Meeting of the Chicago Linguistic Society. Chicago: University of Chicago.

Cormack, A., 1985. "VP Anaphora: Variables and Scope", in F. Landman and F. Veltman (eds.), Varieties of Formal Semantics. Dordrecht: Foris.

Engdahl, E., 1986. Constituent Questions. Dordrecht: Reidel.

Fiengo, R. and R. May, 1994. Indices and Identity. Cambridge: MIT Press (Linguistic Inquiry Monograph Series 24).

Fox, D., 1995. "Economy and Scope", Natural Language Semantics 3.

Groenendijk, J. and M. Stokhof, 1983. "Interrogative Quantifiers and Skolem Functions", in K. Ehlich and H. van Riemsdijk (eds.), Connectedness in Sentence, Discourse and Text.. Tilburg: Tilburg University.

Heim, I., 1997. "Predicates or Formulas? Evidence from Ellipsis", in Proceedings of the 7th Conference on Semantics and Linguistic Theory. Cornell University: Cornell Working Papers in Linguistics.

Jacobson, P., 1989. "A(nother) Categorial Grammar Account of Extraction". Presented at the Conference on Categorial Grammar, LSA Summer Institute, Tucson, Arizona. 
Jacobson, P., 1992a. "Flexible Categorial Grammar: Questions and Prospects", in R. Levine (ed.), Formal Grammar: Theory and Implementation. Oxford: Oxford University Press.

Jacobson, P., 1992b. "Antecedent Contained Deletion in a Variable Free Semantics", in C. Barker and D. Dowty (eds.), Proceedings of the 2nd Conference on Semantics and Linguistic Theory. Ohio State University: OSU Working Papers in Linguistics.

Jacobson, P., 1996. "The Locality of Interpretation: The Case of Binding and Coordination", in Proceedings of the 6th Conference on Semantics and Linguistic Theory. Cornell University: Cornell Working Papers in Linguistics.

Jacobson, P., to appear. "Towards a Variable Free Semantics", Linguistics and Philosophy.

Kennedy, C., 1994. "Argument Contained Ellipsis", ms. University of California, Santa Cruz.

Lappin, S., 1996. "The Interpretation of Ellipsis", in S. Lappin (ed.), The Handbook of Contemporary Semantic Theory. Oxford: Blackwell, Inc.

Larson, R. and R. May, 1990. "Antecedent containment or vacuous movement: Reply to Baltin", Linguistic Inquiry 21.

Oehrle, R., 1990. "Categorial Frameworks, Coordination, and Extraction, in A. Halpern (ed.), Proceedings of the Ninth West Coast Conference on Formal Linguistics. Stanford: CSLI Publications.

Partee, B. and E. Bach, 1981. "Quantification, pronouns, and VP anaphora", in J. Groenendijk, T. Jannsen, and M. Stokhof (eds.), Formal methods in the study of language: Proceedings of the Third Amsterdam Colloquium. Amsterdam: Mathematisch Centrum.

Ross, J.R., 1967. Constraints on Variables in Syntax. Ph.D. Dissertation, MIT.

Rooth, M., 1992. "Ellipsis Redundancy and Reduction Redundancy", in S. Berman and A. Hestvik (eds.), Proceedings of the Stuttgart Ellipsis Workshop. Heidelberg: Arbeitspapiere des Sonderforschungsbereichs 340.

Sag, I., 1976. Deletion and Logical Form. Ph.D. Dissertation, MIT.

Sharvit, Y., 1997. "Against Reconstruction", paper presented at the Jerusalem Semantics Conf erence, Hebrew University of Jerusalem.

Steedman, M., 1987. "Combinatory Grammars and Parasitic Gaps", Natural Language and Linguistic Theory 5.

Williams, E., 1977. "Discourse and Logical Form", Linguistic Inquiry 8. 